\title{
Endoscopic ultrasound with fine-needle aspiration facilitates diagnosis of metastatic iliac lymph node invasion in prostate cancer
}

We have recently published our pilot data on endoscopic ultrasonography (EUS) for staging prostate cancer [1]. EUS with flexible instruments has the additional potential for imaging the iliac region in the search for lymph node metastases with confirmation by EUS-guided fine-needle aspiration (FNA) in prostate cancer; this is an area that cannot be accessed with nonoptical transrectal rigid ultrasonography (TRUS) probes.

A 65-year-old man was referred with an increased prostate-specific antigen (PSA) level of $37.9 \mathrm{ng} / \mathrm{mL}$. An abdominopelvic CT scan was normal. Transrectal EUS was performed to image the prostate and revealed a hypoechoic, round nodule in the peripheral zone of the prostate ( $\bullet$ Fig. 1). Multiple EUS-guided FNA passes were done using a 22-gauge needle (WilsonCook, Winston-Salem, North Carolina, USA). Cytopathological study showed undifferentiated adenocarcinoma. An enlarged iliac lymph node $(13 \mathrm{~mm})$ was seen by EUS by advancing the echoendoscope to the level of the iliac vessels (around 20-25 cm from the anus). Transcolonic EUS-FNA of the left iliac lymph node was done in the same exam (० Figs. 2-4), and histopathology demonstrated metastatic undifferentiated prostate adenocarcinoma ( $\bullet$ Fig. 5).

Staging techniques for prostate cancer include ultrasonography, CT, and magnetic resonance imaging (MRI). Ultrasonography, by the transabdominal, transurethral, transperineal, or transrectal route, allows characterization of the prostate parenchyma. Other techniques for local and nodal staging of prostate cancer include MRI, magnetic resonance spectroscopic imaging (MRSI), dynamic-enhanced MRI, positron emission tomography (PET), endorectal power Doppler ultrasonography, lymphotropic MRI contrast agents, and diffusion MRI [2,3]. EUS may be another useful method for highresolution imaging of prostate cancer [1]. Lymph node staging is an important issue

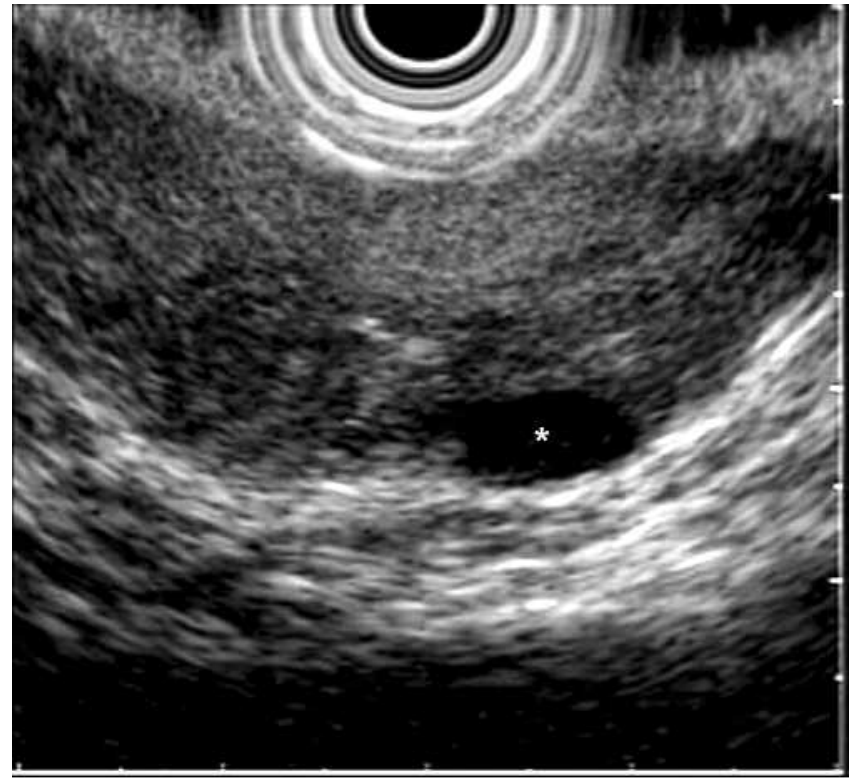

Fig. 1 Transrectal endoscopic ultrasonography (EUS) of the prostate, revealing a hypoechoic, round, and homogeneous nodule in the peripheral zone of the prostate $\left({ }^{*}\right)$.

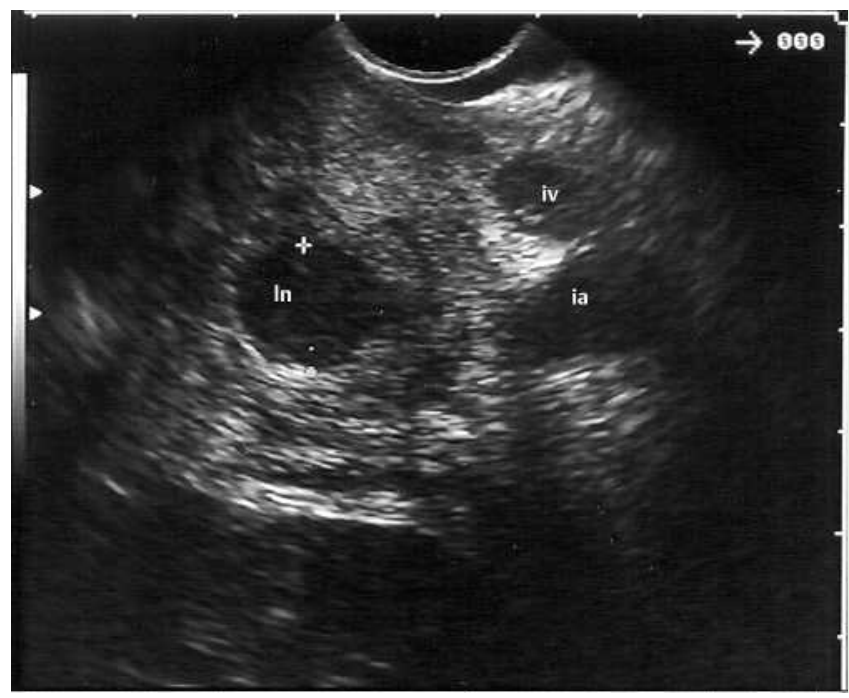

Fig. 2 An enlarged iliac lymph node (In). iv, lliac vein; ia, iliac artery.

in prostate cancer that directly impacts management and outcome. Digital rectal examination and TRUS are not accurate in predicting lymph node metastases [4]. A meta-analysis by Hovels et al. [5] showed a low accuracy of CT and MRI for lymph node staging of prostate cancer. We believe that there is potential for EUS with FNA to play an important, minimally invasive role in lymph node staging of prostate cancer.

Endoscopy_UCTN_Code_TTT_1AS_2AZ Endoscopy_UCTN_Code_TTT_1AS_2AF 

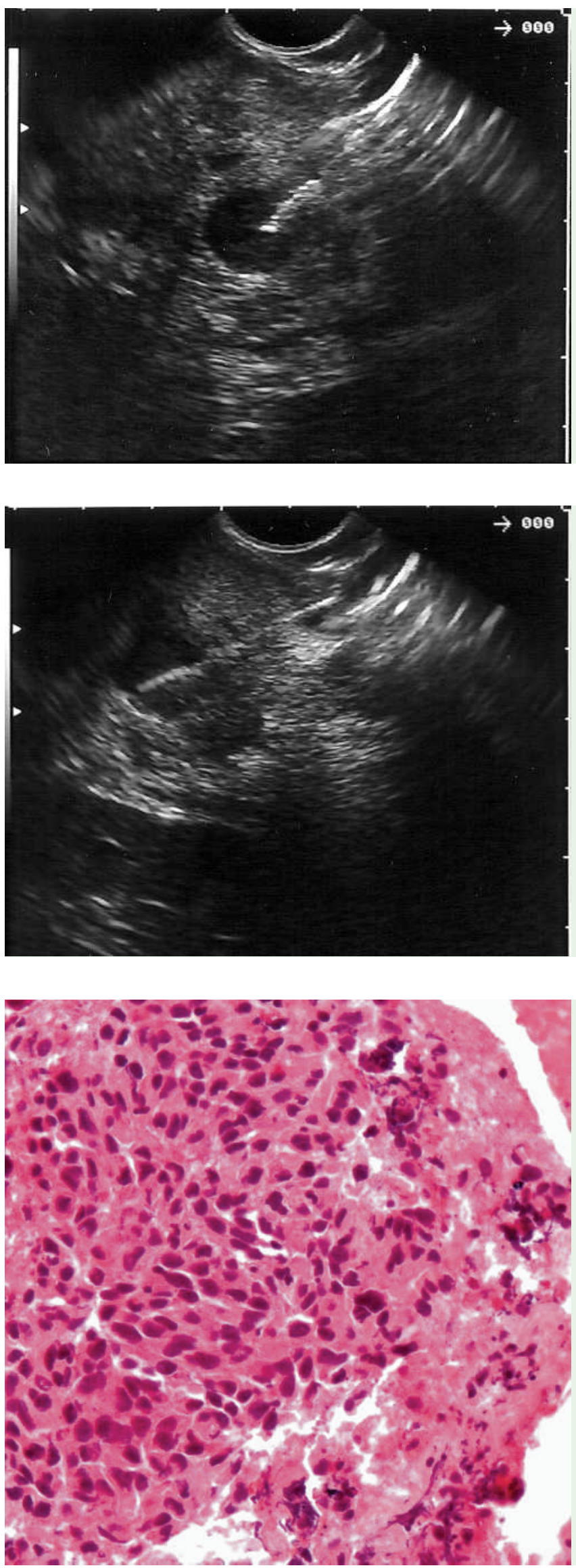

Fig. 3 EUS-guided fine-needle aspiration (EUS-FNA) of the iliac lymph node with the needle in the center of the lymph node.

Fig. 4 EUS-FNA of the iliac lymph node with the needle tip at the periphery of the lymph node.

E. L. A. Artifon ${ }^{1}$, M. Srougi ${ }^{2}$,

A. M. Lucon' ${ }^{2}$, P. Sakai ${ }^{1}$, M. S. Bhutani ${ }^{3}$

1 Gastroenterology Department, University of São Paulo School of Medicine, Sao Paulo, Brazil

2 Urology Department, University of São Paulo School of Medicine, Sao Paulo, Brazil

3 UT MD Anderson Cancer Center, Houston, Texas, USA

\section{References}

1 Artifon EL, Sakai P, Ishioka S, Silva AF et al. EUS for locoregional staging of prostate cancer - a pilot study. Gastrointest Endosc 2007; 65: 440-447

2 Leibovici D, Kamat AM, Do KA et al. Transrectal ultrasound versus magnetic resonance imaging for detection of rectal wall invasion by prostate cancer. Prostate 2005; 62: 101 104

3 Hersh MR, Knapp EL, Choi J. Newer imaging modalities to assess tumor in the prostate. Cancer Control 2004; 11: 353-357

4 Spiess PE, Busby JE, Jordan JJ et al. Can digital rectal examination or transrectal ultrasonography biopsy findings predict the side of nodal metastasis in prostate cancer? Urol Oncol 2008; 26: 25 - 30

5 Hovels AM, Heesakkers RA, Adang EM et al. The diagnostic accuracy of CT and MRI in the staging of pelvic lymph nodes in patients with prostate cancer: a meta-analysis. Clin Radiol 2008; 63: 387-395

Bibliography

DOI $10.1055 / \mathrm{s}-0028-1119477$

Endoscopy 2009; 41: E243-E244

(c) Georg Thieme Verlag KG Stuttgart · New York . ISSN 0013-726X

\section{Corresponding author}

\section{S. Bhutani, MD}

Fig. 5 Cytopathological section from EUSFNA of the iliac lymph node, showing a metastatic adenocarcinoma with cytology similar to that of the prostate FNA.

\section{Department of Gastroenterology,}

Hepatology and Nutrition

Unit 436

UT MD Anderson Cancer Center

Faculty Center Room 10.2028

1515 Holcombe Blvd

Houston

TX 77030-4009

USA

Fax: +1-713-5634398

Manoop.Bhutani@mdanderson.org 